\title{
Théologiques
}

Théologiques

\section{Vie et mort en Afrique noire}

\section{Marcel Anganga}

Volume 19, numéro 1, 2011

Théologie africaine et vie

URI : https://id.erudit.org/iderudit/1014182ar

DOI : https://doi.org/10.7202/1014182ar

Aller au sommaire du numéro

Éditeur(s)

Faculté de théologie et de sciences des religions, Université de Montréal

ISSN

1188-7109 (imprimé)

1492-1413 (numérique)

Découvrir la revue

Citer cet article

Anganga, M. (2011). Vie et mort en Afrique noire. Théologiques, 19(1), 87-106. https://doi.org/10.7202/1014182ar

\section{Résumé de l'article}

En Afrique, continent-Mère de l'homme et source de notre civilisation, vie et mort, depuis plus de 200000 ans avant notre ère, sont liées. Inséparables. Elles constituent, ensemble, les deux faces de l'existence humaine et, par ce fait, la mort se veut la conséquence de la vie. Dès lors, dans la cosmogonie négro-africaine dont les traces sont visibles dans le judaïsme et le christianisme, l'idéologie de la vie prime sur celle de la thanatologie, car la vie ne finit pas avec la mort. A contrario, elle la dépasse, la transcende et continue dans l'Au-delà. Ainsi, la mort n'est pas le dernier mot de la vie pour l'Africain. Celle-ci est, reste et demeurera une phrase en pointillés qui s'achèvera au village des ancêtres lors du retour final.
Ce document est protégé par la loi sur le droit d'auteur. L'utilisation des services d'Érudit (y compris la reproduction) est assujettie à sa politique d'utilisation que vous pouvez consulter en ligne.

https://apropos.erudit.org/fr/usagers/politique-dutilisation/ 


\section{Vie et mort en Afrique noire}

Marcel AngangA

De plus en plus, pour les individus comme pour les sociétés, la mort apparaît comme la clé de l'histoire. La philosophie existentialiste proclame le triomphe de la mort; le matérialisme glouton précipite l'individu des sociétés vers la mort; la littérature, le cinéma, la télévision n'ont plus d'autres leçons à enseigner à l'humanité que l'art de donner la mort. Or l'héritage culturel africain, avec sa vision du monde et sa conception de l'homme, préconise tout juste le contraire. C'est-à-dire la victoire de la vie sur la mort. La civilisation dans le monde pharaonique comme dans l'Afrique traditionnelle consiste à organiser et à gérer l'homme dans le cosmos, en vue d'assurer la victoire de la vie sur la mort. Pour nous, la question est simple. Nous disons qu'en face des idéologies de la mort, il y a place pour les idéologies de la vie et que l'Afrique a quelque chose à apporter dans la recherche, dans l'organisation et dans la gestion de l'homme et du cosmos aujourd'hui. La contribution de l'Afrique doit être celle d'une civilisation du triomphe de la vie sur la mort. (Mveng et Lipawing 1996)

Ces sages paroles du feu Camerounais jésuite Engelbert Mveng, quelques mois avant son assassinat à Yaoundé ${ }^{1}$, constituent le pacte d'écriture du présent article. Elles s'inscrivent, avec pertinence et force perlocu-

* Marcel Anganga est docteur en Écriture Sainte de l'Institut Catholique de Paris et de l'Université Catholique de Leuven en Belgique (2010). Il est curé paroissial des secteurs de Châtillon Coligny et de Nogent-sur-Vernisson dans le diocèse d'Orléans. Ses recherches portent actuellement sur l'évangile selon Saint Marc et sur la relation entre la culture des peuples et l'Écriture Sainte. Il a récemment publié (2011) Analyse narrative du récit johannique de la Passion. Enjeux théologiques pour la célébration $d u$ vendredi Saint, ARNT, Villeneuve d'Ascq et, en collaboration avec François Kabasele et Sylvain Nkongolo, (2011) Naissances insolites en terre africaine, Karthala.

1. «Le matin du 23 avril 1995 le P. E. Mveng fut trouvé mort. Étranglé, couché dans son lit face au plafond, une profonde blessure à la tête. Un meurtre inexplicable, puisque rien n'avait été emporté de sa chambre». Extrait tiré du site web <http:// www.afriquespoir.com/cibles/page6.html> sur le Cameroun, (s.d). 
tionnaire, dans une séculaire confession de l'homme des Tropiques sur sa conception toute particulière de vie et mort. Il s'agit, en effet, en Afrique d'une "conception autre" de cette réalité qui, bien que prioritairement anthropologico-philosophique, sera, dans notre contribution, enrichie et éclairée par des indications tant historiques, culturelles, sociologiques que religieuses.

La question de vie et mort, d'aucuns ne l'ignorent, est humaine. Existentielle. Elle est liée à la provenance de l'homme, à son parcours et à son destin. Se posant et s'imposant d'elle-même, jamais elle n'a cessé, depuis la nuit des temps, de hanter l'humain qu'elle a précédé dans l'existence, à en croire ce dicton bàntu du peuple Lubà du Kàsaayì, au Congo démocratique: "Pànu ìmpasangana pakolè. Masela nè bilùnde ìmbisangana bimenà ", ce qui veut dire: "L'ici-bas est trouvé dur, des hautes herbes et des souches coriaces défiant la houe sont trouvées déjà-là ». Autrement dit, vie et mort, selon la cosmogonie africaine, sont aînées et antérieures à l'homme. Elles ont devancé ce dernier qui, dans la vie, est un puîné subissant au quotidien l'expérience d'enfantement et de trépas, de larmes et de sourire, d'enthousiasme et de chagrin. C'est autour d'elles que gravitent, pour le Mùntu [l'homme], tout son être. Régine Mofila l'exprime en ces termes:

La spiritualité du muntu est centrée sur les concepts «vie et mort». La vie étant une réalité sacrée, elle reste le centre de son existence. De même le concept de mort est intimement lié à la vie. Dans le vécu de tous les jours, la vie est conçue par le muntu comme une lutte engagée contre tout ce qui provoque la mort, bien que celle-ci ne soit qu'un pas vers une vie de l'audelà, une vie avec les ancêtres. Cette façon de concevoir la vie et la mort est un atout des cultures africaines en général et du muntu en particulier. (Mofila 1997, 26)

Dès lors, une réflexion portant sur «vie et mort en Afrique Noire» est une excellente opportunité à saisir. Elle permet, dans un premier temps, de revisiter la vérité scientifique portant sur l'origine de l'homme, mais qui, malencontreusement, se trouve encore, même en ce début du $\mathrm{xxI}^{\mathrm{e}}$ siècle, torpillée, déformée et contestée par certains idéologues au nom de leur "préjugé raciste» (Attolodé 2004, 4). Deuxièmement, elle autorise à réaffirmer, à la suite des travaux probants, l'incontournable place qu'occupe le continent noir dans l'évolution de l'humanité, malgré les guerres interminables qui lui sont imposées, l'occupation de ses terres ainsi que l'exploitation de ses richesses par les grands prédateurs de ce monde. Enfin, l'occasion est propice pour évaluer et repenser, à nouveaux frais, le rôle et l'apport de l'Afrique à la compréhension de la réalité vie et mort face aux 
diverses conceptions négativistes et destructrices sévissant dans le monde. Mais, à partir de quel «lieu» doit-on débattre ce sujet?

\section{D'où faut-il, «objectivement parlant», aborder la question de vie et mort?}

La problématique de vie et mort, parce qu'elle concerne en premier chef l'humain, s'inscrit dans le registre diachronique. À ce titre, l'approcher à partir des visions occidentale (grecque, romaine, etc.), asiatique (par référence à Jésus ${ }^{2}$ ) ou judéo-chrétienne comme il est habituel de le faire, c'est malheureusement rejeter sciemment les acquis importants des sciences humaines. C'est falsifier l'histoire. C'est non seulement, selon les propos du chercheur et historien africain Cheik Anta Diop (1984), "raconter l'histoire à rebours et vouloir renverser le courant de l'évolution " mais c'est aussi refuser, incontestablement, au nom d'un certain à priori à la fois intentionnel et pitoyable, la déconcertante et objective vérité des faits à la suite de l'Allemand $\mathrm{Hegel}^{3}$, du Français Lévy-Bruhl, de l'Écossais David Hume, ainsi que de leurs épigones au préjugé identique. À la fois intellec-

2. "Jésus est né, a vécu, a prêché et est mort en Asie. Cependant, on le considère souvent comme un Occidental. Historiquement parlant, la chrétienté s'est plus largement répandue vers l'Ouest que l'Est. [Bien entendu, les termes "Ouest" et "Est" sont utilisés en rapport avec la Palestine où Jésus est né et a vécu]. Cette étendue géographique a coïncidé avec l'étendue de l'Empire romain influencée par la culture grecque et le système romain politique judiciaire. » (Amaladoss 2007, 9).

3. Hegel a prêché dans ses Leçons sur la philosophie de l'Histoire que le « Nègre est sans culture ", d'où son irrémédiable inertie. Ainsi, n'ayant ni raison ni liberté, le Noir est, selon Hegel, «hors de l'histoire». Tristes paroles que reprendra à Dakar, dans son discours du 26/07/2007, Nicolas Sarkozy, le président français qui n’a pas pu citer sa source ni continuer cette autre affirmation gratuite de son maître Hegel: "L'Afrique, aussi loin que remonte l'histoire, est restée fermée, sans lien avec le reste du monde; c'est le pays de l'or, replié sur lui-même, le pays de l'enfance qui, au-delà du jour de l'histoire consciente, est enveloppé dans la couleur noire de la nuit [...] c'est un monde anhistorique non développé, entièrement prisonnier de l'esprit naturel et dont la place se trouve encore au seuil de l'histoire universelle». C'est pourquoi, poursuit Hegel, «Le Nègre représente l'homme naturel dans toute sa barbarie et son absence de discipline.» (1979, 247, 251 et 269). Pour sa part, David Hume écrit: «Je suspecte les Nègres et en général les autres espèces humaines d'être naturellement inférieures à la race blanche. Il n'y a jamais eu de nation civilisée d'une autre couleur que la couleur blanche ni d'individu illustre par ses actions ou par sa capacité de réflexion [...] Il n'y a chez eux ni engins manufacturés, ni art, ni science. Sans faire mention de nos colonies, il y a des nègres esclaves dispersés à travers l'Europe; on n'a jamais découvert chez eux le moindre signe d'intelligence.» (Hume 1852, 252) 
tuels, savants et civilisés, ces penseurs n'avaient pas à l'esprit que "devant les faits, [dit-on], point de preuves». Assurément, si les données scientifiques modernes confirment que l'Afrique est le berceau de l'humanité, ne serait-il pas équitable, comme l'indiquent Richard Leakey et Ian Mc Dougall, «que les concepts spirituels que l'on trouve dans les différentes religions du monde, aient été élaborés par les Africains en premier, sachant que les humains modernes sont nés en Afrique, 200000 ans avant notre ère "? (Cité par Ogowè Nyalendo 2010, 1).

Évidemment, s'il est avéré qu'à une époque très reculée, les habitants de la vallée du Nil se sont questionnés sur le sens de la vie et de la mort ainsi que sur l'idée de l'implication des actes que l'homme a posés au cours de sa vie terrestre, c'est donc sur les terres africaines qu'a eu lieu, comme l'atteste Jacques Lacarrière, écrivain français et spécialiste de la Grèce antique, la plus vieille représentation portant sur vie et mort. Rendonsnous à l'évidence:

La plus ancienne idée d'un jugement des âmes, destiné à leur conférer un sort différent selon leurs mérites, est née sur les rives du Nil. On n'en trouve aucune trace dans la mythologie sumérienne ni akkadienne mais en Égypte, [...] la légende d'Osiris, en ouvrant à chacun les portes d'un monde régénéré et en promettant la résurrection, impliquait la notion d'un jugement, d'un tribunal, bref d'une «sélection » des âmes appelées à connaître le même sort qu'Osiris. D'ailleurs, Osiris lui-même, une fois ressuscité, n'avait-il pas à se défendre contre les attaques de Seth et faire reconnaître son innocence par le tribunal des grands dieux? L'homme, à l'image d'Osiris, devait donc lui aussi faire admettre son innocence par un tribunal identique, tribunal dont le Livre des morts nous a laissé une description impressionnante. » (Lacarrière 1998, 471-472)

Cela étant et, conséquemment, ne serait-il pas intègre, honnêteté scientifique oblige, d'éclairer la lanterne de l'humanité — sur la question de vie et mort - par l'entremise de la conception du premier peuple qui est apparu sur la terre, c'est-à-dire l'Africain ? Dès lors, l'Afrique s'imposant, naturellement, en lieu-aîné à la fois culturel et géographique qui a porté l'homme moderne (Braüer 1994, 132-150) à sa naissance constitue, comme le confirme Christiane Noblecourt d'heureuse mémoire ${ }^{4}$, «la source de la culture moderne». Elle note:

4. Née le 17 novembre 1913, elle a tiré sa révérence le 26 juin 2011, en France, à l'âge de 97 ans. 
Mon propos, en écrivant ce livre, est d'introduire mes lecteurs, sans leur infliger de savantes explications, ni les fatiguer par un verbe pompeux, à la découverte des thèmes fondamentaux sur lesquels notre civilisation s'est construite. L'Égypte ancienne leur apparaîtra alors comme une pionnière en raison des connaissances, de la sagesse et de l'humanisme qu'elle nous a transmis. Elle demeure la grande inspiratrice pour ceux qui désirent retrouver leurs racines. (Noblecourt 2006, 6)

Cette certification subversive se trouve renforcée par un commentaire de l'ouvrage, rédigé par Brigitte Kermel et placé sur la $4^{\text {e }}$ de couverture. En voici un extrait:

Quel est le point commun entre le jeu de l'oie, l'alphabet, le calendrier, les animaux des fables d'Ésope et de La Fontaine, le test de grossesse, les traitements contre la migraine ou encore les châteaux forts? Leur origine prend sa source au cœur de l'Égypte ancienne. Philosophie, médecine, techniques et sciences, théologie... ces disciplines fondatrices nous viennent, toutes, en ligne droite, des 4000 ans d'histoire de la civilisation égyptienne. Christiane Desroches Noblecourt, la plus respectée et la plus audacieuse des égyptologues contemporaines, dresse un panorama étourdissant du legs insoupçonné de l'Égypte ancienne à l'Occident, dans sa vie quotidienne comme dans ses fondements religieux et philosophiques les plus essentiels. Une démonstration aussi limpide que passionnante qui nous incite à tourner plus que jamais nos regards vers une civilisation incroyablement féconde, indéniablement liée à la naissance de la nôtre.

Ce n'est pas tout. "L'antériorité de la longue tradition négroégyptienne est soulignée ainsi que son influence sur les peuples sémitiques et indo-européens attestée par son empreinte laissée dans le judaïsme et le christianisme ", selon le témoignage du professeur Théophile Obenga (1997-1998, 9-53). De même que les nations nègres, ainsi que leurs cultures, sont antérieures à la civilisation égypto-nubienne, celle d'Égypte fait ellemême partie de l'univers nègre (Diop 1967). Par conséquent: «N'est-il pas de plus en plus attesté chez les historiens que le judaïsme et le christianisme n'ont rien inventé des figures majeures qu'ils prodiguent, et qu'au contraire, ils les ont puisées dans le trésor culturel ancien des autres peuples, notamment de l'Égypte et de la Mésopotamie ? (Kabasele, Nkongolo et Anganga 2010, 8). Cela est d'autant plus vrai que «L'Égypte pharaonique était une civilisation nilotique, née, épanouie et morte aux bords du Nil, dans la vallée du Bas-Nil. Et de ce fait, ses racines appartiennent totalement à l'univers culturel négro-africain (Érythrée, Abyssinie, Éthiopie, Nubie, ancien Soudan, Sahara préhistorique, etc.)»(Omotunde 2000, 40-41; 
nous soulignons). D'où l'interrogation fondamentale suivante de François Kabasele $(2005,223)$ : "L'Afrique noire n'a-t-elle pas une part prépondérante dans l'héritage spirituel de l'Égypte ancienne? Et s'il en est ainsi, les écrits juifs, bien que postérieurs aux textes sacrés des pyramides, ne seraient-ils pas inspirés des traditions égyptiennes?»

Ces préalables indiquent et justifient, comme on peut le constater, que l'Afrique, au titre de "Mère de la vie ", est l'unique lieu autorisé à partir duquel devrait partir tout discours, si pluridisciplinaire soit-il, portant sur vie et mort. Ainsi, la question à laquelle nous voulons répondre est la suivante: quelles sont, à partir des données objectives, les approches culturelles, les configurations et les significations que le couple vie et mort a revêtues en Afrique, berceau de l'humanité, au cours des âges? Pour le dire autrement, comment l'Afrique, par son antériorité à toutes les autres civilisations, peut-elle apporter au monde une autre philosophie, une manière différente d'être, eu égard aux diverses «stratégies et implications idéologiques, narratologiques et énonciatives " (Amougou 2008, 1) sur le binôme vie et mort? Mais cherchons, avant d'aller plus loin, cette conception africaine de l'homme et en quoi elle diverge de celles d'autres civilisations.

\section{La conception de l'homme dans les plus vieilles philosophies}

Comme nous l'avons précédemment illustré, il est inexact d'affirmer, dans l'aujourd'hui, que les cultures grecques et judéo-chrétiennes ordinairement présentées comme les plus anciennes seraient fondatrices des concepts et idées qu'elles prônent. Elles sont, a contrario et preuves à l'appui, tributaires de la pensée africaine ${ }^{5}$.

Sur le plan anthropologique, la pensée grecque reste dominée, avec ses deux tenants classiques que sont Platon et Aristote, par un dualisme radical avec la prédominance de l'âme sur le corps. Pour le premier, sous l'influence de la tradition religieuse de croyance orphico-pythagoricienne,

5. «[...] et c'est encore grâce à l'Égypte en plein déclin, que Thalès introduisit la conception de l'immortalité de l'âme; Pythagore après plus de vingt années d'études en Égypte, fut initié aux mystères; Platon (sur la Philosophie) dans ces œuvres, Timée (Platon utilise sans le citer toutes les idées égyptiennes à savoir: la composition du monde, l'immortalité de l'âme, les quatre éléments "feu, terre, air, eau", la notion du temps, essence mathématique du monde conçue comme nombre pur...), le Phèdre, et le Phédon fait référence à l' Égypte». Voir SHENOC (2008), "La conception des peuples noirs de la mort et de l'immortalité », <http://www.shenoc.com/la_conception_des_peuples_noirs_de_la_mort_et_de_l-immortalite.htm>, en référence à Obenga (1984). 
l'âme est dans le corps comme dans une enveloppe charnelle, dans une prison ou encore dans un tombeau. C'est à ce titre que l'âme fait l'homme ${ }^{6}$ parce qu'elle commande le corps. Quant au second (Maison 1990, 1174), par sa théorie de l'hylémorphisme, il prône l'indéfectible lien des deux principes la forme et la matière qui, appliqués à l'anthropologie, révèlent que «l'âme humaine soit un genre d'âme tout différent, c'est-à-dire intellective», parce que possédant, comme caractéristiques propres, "l'intelligence et la faculté théorétique » contrairement à l'âme végétative et à l'âme sensitive (Aristote, De Anima 1934, 76-77). Point n'est besoin de rappeler que cette vision grecque de disséquer l'être humain en âme et corps n'a pas épargné le christianisme, alors que dans les pays des missions, l'âme de l'évangélisé valait plus que son corps. D'où la légitimation de la théologie missionnaire du salut des âmes.

Pour sa part, la conception sémite de l'homme, d'où est née la judéochrétienne, contrairement à celle des Grecs, ne conçoit pas l'homme comme distinctement composé de corps et de l'âme. Sont impensables une quelconque préexistence de l'âme au corps et une dualité substantielle entre les deux; car, selon le philosophe helléniste et hébraïsant exégète français Claude Tresmontant, «l'homme est créé une âme vivante », comme l'atteste la LXX ${ }^{7}$. Ainsi, pour les Hébreux, il est un non-sens de concevoir un corps sans âme au sens grec du terme. D'ailleurs, ce fut une grave erreur des Grecs

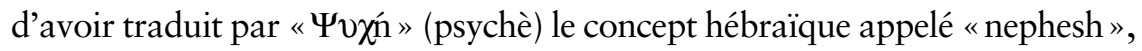
ou principe vital, malheureusement rendu par "anima» en latin et par «âme» en français. Or, selon la tradition sémite, l'homme intégral est désigné par «basar». Claude Tresmontant l'authentifie quand il écrit:

En hébreu, la basar, ce n'est pas le corps en tant que distinct de l'âme. La basar, c'est la totalité humaine, pour nous français "corps et âme ", la totalité psycho-physiologique, ou psycho-somatique. C'est le biologique et le physiologique à la fois. L'hébreu basar ne correspond donc nullement à ce que nous appelons le «corps", ni «la chair» distincte de l'âme, mais à ce que nous appelons l'homme vivant. (Tresmontant 1971, 62)

Cela est d'autant plus vrai que pour l'anthropologue français Jean Mouroux, «La personne est donc un esprit à la fois immergé et émergent, immanent et transcendant au corps. Cet esprit et ce corps ne sont qu'un

6. Voir Alcibiade 129a-131d: «Et Socrate de préciser à Alcibiade: “C'est l'âme qui est l'homme"."

7. Tresmontant $(1971,59-60)$ : «Yhwh forma l'homme de la poussière du sol, et il souffla dans ses narines un souffle de vie, et l'homme devint une âme vivante (Gn 2).» 
être» (1943, 106-107; voir Gn 2,7; 1Th 5,23). Du point de vue critique, on ne peut en douter, l'anthropologie asiatique de l'unité psycho-somatique dont le judaïsme se fait messager n'est pas qu'en rupture avec celle des hellénisants, mais elle est reprise par le christianisme alors qu'elle fonde son origine dans la plus vieille des visions du monde, c'est-à-dire l'Afrique Noire, terre ancestrale de l'humain.

Dès lors, étant donné que «communiquer est éminemment culturel », l'anthropologie africaine, en l'occurrence celle des Bàntu (Mujinya Nimisi, 1978), partant de son langage, de ses représentations, de ses codes et de son matériel symbolique définit l'homme, à la différence de la conception grecque, comme un tout. Un ensemble. Quelques concepts méritent d'être clarifiés.

En premier, la notion de l'être. Elle est commune à chaque élément présent dans la nature. Dès lors, le monde est conçu en Afrique, chez les Bàntu, «comme un tout organique, dont tous les éléments sont réunis par un dénominateur commun qui est la vie, valeur suprême vers laquelle tout tend» (Kabasele 2005, 259). Ainsi, la vie est commune à tout ce qui vit, qui fait partie de la nature créée et qui participe, à de degrés divers, à cet ordre. François Kabasele le décrit dans l'extrait suivant:

Tous les êtres sont participants à cette vie dont la source première est Dieu. Cela veut dire que l'herbe a la vie, l'eau a la vie, l'animal a la vie, la motte de terre a la vie, les étoiles et les astres ont la vie. On l'éprouve par la force qui se dégage d'eux et qui se déploie autour d'eux: ainsi la terre fait germer les graines; l'eau étanche la soif; l'herbe peut nourrir ou tuer quand on la consomme; l'herbe peut tuer le microbe et guérir un malade; le soleil réveille les hommes, fait croître, réchauffe en chassant le froid; la lune fait varier les tempéraments, provoque des changements dans le corps humain; chez l'homme, la vie se déploie d'une manière encore plus rapide: il entre en relations, il produit, il réfléchit, il engendre... La participation à la vie se fait à de degrés divers et permet ainsi une interdépendance multiforme entre les êtres, selon leur nature et leur affinité: les Ancêtres sur les descendants, les Forts (chefs, guérisseurs, sorciers) sur les mouvements de la nature et des hommes, les Aînés sur les puînés, les parents sur leurs enfants, ceux qui sont dans leurs droits (ayant le lusanzu), sur les fautifs, etc. (Kabasele Lumbala 2005, 259-260)

Comme deuxième concept nous évoquons le Bumùùntù ou «la valeur, l'être de l'homme». Celui-ci a pour sens qu' «en chaque chose est une autre chose; dans chaque homme ou personne se trouve un petit homme, c'està-dire qu'il y a lieu de distinguer dans l'être matériel ce qui tombe sous le 
sens, ou phénomène apparent, de ce qui ne se voit pas, ou nature intrinsèque de l'être: l'homme (corps), l'ombre, le souffle (signe apparent de la vie), et l'homme lui-même ou l'âme" (Tempels 1945, 37 ; voir aussi Mujinya Nimisi, 1978). C'est l'ensemble de ces éléments qui font l'homme. Pas un sans l'autre. C'est ce discernement et cette logique que Placide Tempels, missionnaire catholique belge et pionnier de la philosophie africaine, a découverts dans l'ontologie du Sud dont la «Vie», valeur absolue, est le fondement. Ayant pour source Dieu, la vie, également nommée force vitale, chez le Mùntu, se reçoit et s'acquiert. Elle exige renforcement, protection et croissance en cas de menace ou de diminution. D'où la pratique des rites traversant toute la vie de l'Africain ainsi que les prières qu'il adresse à la fois aux Ancêtres et à Dieu, l'Être Suprême. Tempels le certifie quand il écrit :

La force, la vie puissante, l'énergie vitale sont l'objet des prières et des invocations à Dieu, aux esprits et aux défunts, ainsi que de tout ce qu'on est convenu de nommer "magie", "divination» et "remèdes magiques » ou plutôt des forces raffermissements de la nature. Eux-mêmes diront qu'ils s'adressent au "devin » pour apprendre "des paroles de vie », qu'il enseigne la manière de renforcer la vie. Dans chaque langage bantou on découvrira facilement des mots ou locutions désignant une force, qui n'est pas exclusivement «corporelle», mais «totalement humaine». Ils parlent de la force de notre être entier, de toute notre vie. Leurs paroles désignent «l'intégrité » de l'être. Le bwanga (ce qu'on traduit par remède magique) ne doit pas nécessairement, d'après eux, être appliqué ou collé à la plaie ou au membre malade. Il n'a pas en premier lieu un effet thérapeutique local, mais il renforce, il augmente directement la force vitale, ou l'être même. (Tempels $1945,29)$

Dès cet instant, la relation entre ces êtres ne se traduit pas en terme de conquête par le possessif: "j'ai mon cheval, j'ai ma femme, etc. »; ce qui équivaudrait à une soumission, à un esclavagisme ou à une exploitation de l'autre être. A contrario, le lien entre êtres s'exprime par «l'être-avec». C'est-à-dire qu'on n'est pas seul dans la nature. Chacun des créés ayant une mission à accomplir avec l'autre et à côté de l'autre a le droit d'être respecté. À cet effet, la relation d'interdépendance entre êtres se traduira, selon la linguistique africaine, par l'expression "je suis avec mon mari »; "je suis avec ma femme"; «je suis avec ma chèvre ou je suis avec mon pognon », et non "j'ai ma femme» comme en langue française. Ce mode opératoire exclut toute idée de dominer l'autre, de le rendre dépendant de nous, alors qu'il a sa propre identité - laquelle est, à son tour, liée à la 
nôtre et en appelle à de sains rapports. Cet "être-avec ", comme nous pouvons le constater, rend visible toute une philosophie, un comportement, une mentalité. Il traduit le respect de l'être avec qui on est en relation; il le conçoit non pas comme un objet dont on peut user comme bon nous semble, mais comme celui qu'on doit traiter avec déférence, car notre vie dépend également de son être-là, à nos côtés et avec nous - et nous sommes ensemble-avec-lui dans le monde. C'est dans ce registre que s'inscrit la notion de l'être qui a pour point d'ancrage la force vitale en tant que valeur suprême et centre de la vie chez les Bàntu (voir Tempels 1945, 28-30).

Sous cet éclairage, la «vie» en Afrique est sacrée. Énigmatique. À ce titre, elle ne doit être ni ôtée ni violentée, contrairement à ce qui, malheureusement, s'expérimente au quotidien. C'est pourquoi, dans la tradition, le cadavre de quiconque s'étant suicidé était publiquement fouetté et châtié avant sa mise en terre. Dans cette optique, les guerres, les meurtres, la violence... bref, tout ce qui portait atteinte à la vie humaine était non seulement condamné, mais ostensiblement réprimé et conjuré comme un mauvais sort. Dans le même ordre d'idées, un guérisseur ne pouvait arracher l'écorce d'un arbre ou le feuillage d'une plante, pour préparer une potion, un fétiche ou un médicament pour soigner, sans avoir parlementé avec l'arbre ou la plante en lui adressant, par la parole, sa demande. Ainsi l'Africain est conscient de la dépendance de sa vie humaine de celle d'autres créés qui sont ses adjuvants: faune, végétaux, minerais, inanimés, etc. La vie est, dans ce cadre, un tissu organique, un réseau de relations à des niveaux divers: relation avec l'Être Suprême appelé mâyi mfùkya mukèlè, c'est-à-dire "l'Eau qui a créé le sel », Lui, le Dieu Puissant et "générateur de la force de toute créature» (Tempels 1945, 29); relations avec les Ancêtres qui, en premier, ont eu l'appel et la révélation de Dieu dans leur cheminement, l'ont servi et siègent désormais dans le kaalà kakòmba, village des Ancêtres; relations avec tous les autres intermédiaires visibles et invisibles ainsi que les humains, sans oublier la nature avec laquelle il faut être en harmonie pour renforcer sa vitalité. Bref, en Afrique tout créé est «vie » et participe à la vie: une vie souhaitée, désirée et entretenue. Raison pour laquelle au pays Balubà, c'est par l'expression muoyo ou «la vie» qu'on se salue souhaitant ainsi la vie à la personne à qui l'on dit bonjour.

En somme, le corps, en anthropologie africaine, n'est pas une partie de l'homme, mais l'homme qui s'extériorise. Dans cet homme il y a la force vivante, «la force» ou l'être qui, possédant la vie véritable et pleine, est la force éminente parmi les autres êtres créés. La force et l'être étant intrinsè- 
quement liés, ils le sont aussi dans leur définition, à savoir l'être est force. La copule est à sa place; elle identifie les deux éléments et sanctionne leur jointure. C'est par elle que tient la charpente de toute cette ontologie. Cette force n'est pas, comme chez les autres, un accident. Elle n'est pas non plus physique, mais elle est en revanche, l'essence même de l'être en soi; elle est l'être tel qu'il se manifeste, c'est-à-dire dans sa totalité réelle. L'unicité de la personne humaine est ici affirmée et démontrée.

\section{Contribution de l'Afrique face aux idéologies thanatologiques}

Au regard de l'anthropologie philosophique, l'idéologie funéraire africaine est culturellement inscrite dans les mythes, les contes, proverbes et représentations symboliques de l'Au-delà: en Égypte ancienne, par le Phénix (oiseau fabuleux renaissant toujours de ses cendres en signe de résurrection); en Afrique Noire par un long voyage, la roue de la vie, le pont du jugement et diverses autres images. Au sujet de ces mythes qui véhiculent l'idéologie de mort en Afrique, Dominique Zahan note:

Ces mythes ont des thèmes variés qui justifient l'entrée dans le monde des humains, avec un raisonnement particulier lié à l'immortalité de l'être humain. Un certain nombre de ces récits sont appelés par certains auteurs "le message manqué » (Baumann 1936, 238 ; voir aussi Abrahamsson 111,4). Selon ce thème, la divinité décide d'envoyer aux hommes deux messages, l'un de la mortalité, l'autre de l'immortalité. C'est le premier arrivé à destination qui décide, une fois pour toutes, de la destinée de l'humanité. Dans la plupart de cas, Dieu confie le message de l'immortalité à un animal lent, tandis qu'un animal rapide est chargé de porter le message de l'immortalité. (Zahan 1979, 41)

Dans ce débat idéologique de thanatologie, alors que les énoncés scientifiques et bien d'autres encore, sous l'influence des conceptions diachroniquement ultérieures (philosophie grecque, monde biblique et vision judéo-chrétienne), portent sur la nature et l'explication du «pourquoi » de la mort, démarche qui engage la quête du responsable de la mort ou de sa cause — sans plus —, l'approche philosophique de l'Afrique Noire, en revanche, va au-delà et s'intéresse, quant à elle, au sens de la mort en cherchant à répondre à la question du «comment». Dès lors, ce principe, à la fois philosophique et herméneutique, se trouve au fondement de toute la sagesse et de la doctrine de la mort dans les Tropiques comme nous l'indiquent les dictons suivants: "La mort, est le fruit de la vie. La vie est le fruit de la mort» au pays Diola du Sénégal; «Lufù, ndukùlù, bwanga 
ncidingishilu», c'est-à-dire: «la mort est l'aînée, alors que le fétiche reste un trompe l'œil » chez le Lubà du Kàsaayì, au Congo R.D.; et selon un dicton Sakata: «le vivant sur terre qui ne pense pas à sa mort est un insensé ».

Dans cette perspective, en Afrique, vie et mort sont toutes "données » à l'être humain par le créateur.

Elles sont les connotations fondamentales de l'existence et se trouvent si intimement liées que l'une ne peut pas se concevoir sans l'autre... [et] la mort semble la conséquence inévitable de la vie. C'est dans le contexte d'une telle dialectique que s'inscrivent presque tous les mythes africains d'origine de la mort, depuis longtemps analysés et classés par l'érudition occidentale. (Zahan 1979, 41)

Au regard de cette donnée basique, les conceptions sur la mort divergent selon les cultures, les peuples et les visions du monde. Et dans le cas qui nous concerne, notamment celui de l'Afrique, la conception sur vie et mort se polarise autour des quelques axes majeurs.

Le premier de ces axes est la conviction selon laquelle, «les morts en Afrique ne sont pas morts ». Ils ne sont pas partis, à en croire le poète sénégalais Birago Diop ${ }^{8}$, bien que vivant ailleurs d'une autre façon, ils restent cependant présents parmi les vivants. Ils sont avec eux, mais autrement. Invoqués en cas de nécessité, ils répondent et donnent satisfaction. D'où les rites d'invocations et d'offrandes pratiqués à leur égard. Donc, lors d'une catastrophe naturelle, d'une pandémie en famille ou dans un village, d'un malheur, d'une recherche de bénédiction ou d'autres grâces, l'Africain appellera son grand-père, son père ou sa mère pour le protéger. On parlerait mieux ici d'une médiation plutôt que de superstition, comme l'ont prétendu, malheureusement et sans discernement, nombre des fanatiques chrétiens formatés à la cartésienne. Or, c'est cette même croyance qui se trouve renforcée de manière particulière dans le culte des saints dans la Tradition de l'Église. Sinon ce culte, parce que célébrant des personnages étrangers à l'Africain dont il porte cependant les prénoms même si ceux-ci sont extérieurs à son identité (Diagne 2000, 44-53) — comme si un prénom

8. Voir Les Souffles, poème de Birago expliqué et commenté par Magnier (1990, 223). Selon un autre commentaire: "Pour Léopold Sedar-Senghor, dans la préface des Contes d'Amadou Koumba, Birago Diop nous rappelle ce Pacte lourd mais essentiel qui nous lie à la vie: "Birago Diop ne fait que traduire, à travers la loi de l'interaction des forces vitales, la dialectique de la vie qui est celle de l'univers. À l'anarchie et à la mort s'oppose l'ordre de la vie.” " 
ne pouvait être qu'européen -, constituerait une grande superstition de l'Église et dans l'Église. Dès lors, «Pour saisir et vivre d'emblée le message pascal, c'est-à-dire la mort et la résurrection du Christ, nous pouvons et nous devons donc en tant qu'Africains, nous référer à notre héritage ancestral » clame la sœur congolaise Régine Mofila (1997, 24), étant donné que «Dieu source de tout ce qu'il y a de positif dans notre héritage culturel est en même temps l'auteur de la révélation chrétienne. Fidèle à lui-même, il ne peut se contredire, car à travers les siècles, Dieu en père providentiel a préparé la voie de Évangile» (Mulago 1973, 153). Ainsi Dieu, depuis toute éternité, aurait préparé, en son Fils venu accomplir et parfaire toute culture, les cultures de tous les peuples de la terre pour mieux l'accueillir. C'est cette conviction que nous exprimions dans notre récente publication:

Dieu ne se communique pas en dehors de l'homme et de son environnement, il ne se révèle ni hors de l'homme ni en dehors de ce qui le constitue et le définit. En revanche, Dieu rejoint l'humain dans sa culture, plus exactement «là où il est situé culturellement ». [...] Il ne parle que dans la langue connue de lui, sa propre langue, celle qui lui vient de sa culture et de ses ancêtres. Dieu communique avec l'homme par les diverses expressions du terroir dont celuici est issu: symboles, images, gestes, signes, là où l'homme habite. Ainsi, la culture de chaque peuple dans son ensemble constitue le lieu «de la révélation de Dieu », «de sa présence ", et "de son être-là ». (Anganga 2011, 247)

Comme deuxième axe de l'apport de l'Afrique au monde face à l'idéologie de la mort, nous mentionnons cette sagesse des Tropiques qui certifie que la mort est un passage vers la vie et la vie est un passage vers la mort. En effet, «la vie n'est qu'un passage, le pays des morts étant celui d'où tout homme vient et où tout homme retournera. L'existence alors transcende la vie temporelle, dans une conception cyclique, où l'on ne meurt que pour survivre» (Thomas 1984, 746). Dans cet entendement, la vie de l'homme est composée de deux frontières: la naissance ou la vie perceptible en amont; et la mort au titre de borne existentielle d'en bas. Les deux bornes, comme on le sait, limitent et encadrent l'existence humaine. Elles s'interpellent, s'appellent et interagissent. Cependant, la tension de la vie vers la mort n'est pas à entendre en terme de déchéance ni de destruction car, le vocabulaire usuel de "j'ai perdu mon père " pour dire qu'il est mort, n'est ni propre ni approprié à l'Africain. Une perte induit au dégât, au préjudice, bref au mal, à la détérioration. Le vocable en usage pour parler de la mort, dans les forêts et brousses africaines, depuis la nuit des temps est celui du voyage, d'une excursion. Car il s'imprime dans ce déplacement l'idée d'un retour, non pas sur terre, mais à nos origines, d'où nous sommes tous 
venus. Dans cette optique, l'humain n'est qu'un immigré. Un être en perpétuelle pérégrination. Par sa venue au monde, l'homme, dans la mentalité de l'Africain, est censé quitter ses origines; et par sa mort, il retourne à ces mêmes origines.

Venons-en au troisième pivot qui est celui d'une vision optimiste de la vie. Certes, en Afrique, selon les travaux de l'anthropologue Louis-Vincent Thomas, «la vie et la mort ne sont pas les deux pôles antinomiques du cheminement humain, mais, au contraire, deux réalités qui interfèrent, se complètent et se nourrissent réciproquement» (Thomas 1984, 746). Cette conception positive de la vie est issue des Religions Traditionnelles Africaines où se trouve affirmé, selon les spécialistes, "le caractère inépuisable des forces cosmiques et de la perpétuité de la vie », étant donné qu'en Afrique, le principe vital de l'individu n'est pas détruit par la mort. Dès lors, cet optimisme constitue une véritable grille de lecture de la réalité vie et mort, alors que la civilisation occidentale, bien que de haute technicité, n'a jamais su, au cours de son histoire, l'intégrer à sa réflexion anthropologique. Évoquons une fois de plus Louis-Vincent Thomas qui décortique mieux le sujet.

Car, en Afrique, rien n'est fini et rien n'est commencement, la naissance est une «mort» dans le royaume de l'au-delà d'où l'on est censé venir, ma mort est une "naissance» au monde des ancêtres, monde qui préside à la subsistance et à la vie quotidienne des mortels et qui lui apporte protection et conseil. Car elle n'est jamais une rupture dans ces sociétés où le culte des ancêtres vient tisser des liens constamment resserrés par le sacrifice et la prière, mais aussi par la divinisation et l'écoute du message ancestral, source permanente de cette protection. La mort, au contraire, introduit le défunt au rang d'ancêtre, dont l'esprit renaîtra un jour pour insuffler une nouvelle vie dans l'un de ses descendants. (Thomas 1984, 748)

De cet extrait réaliste, réajustons cependant et précisons que la mort n'introduit pas tout défunt "au rang d'ancêtre ", comme l'affirme son auteur. Sinon tous les défunts africains seraient des ancêtres. Elle l'introduit plutôt dans le village des ancêtres où tous n'ont pas le statut d'ancêtre. Évidemment, n'est considéré en Afrique comme ancêtre, après sa mort, que celui qui a bien vécu; qui a été un exemple et un modèle dans la société; celui a respecté le droit, la justice et l'humain; celui qui, de son vivant, n'a pas pris femme d'autrui, ni volé ni tué; celui qui a traversé ses jours en ayant comme couche la charte traditionnelle, etc. Celui-là peut, au regard de la tradition, être vénéré comme ancêtre. À ce titre, il rejoint l'ordre d'intermédiaires de médiation. Dans cette perspective, il s'avère que vie et 
mort ne se laissent pas séparer en Afrique. Elles sont les deux faces de l'existence. Elles conditionnent le comportement humain; et la mort, frontière d'en bas et dernière expérience de "quittement ", ouvre sur le village des Ancêtres et s'impose comme un des moments clés du cycle vital. Même alors, disons-le, la mort n'est pas, dans le mental et subconscient des Tropiques, le dernier mot de la vie humaine. Celle-ci, en revanche, est et restera, tant que sous les Tropiques le jour se lève et se lèvera, une phrase en pointillés; un texte inachevé dont le terme ne se réalisera que lors du passage ou de la traversée du fleuve qui conduit vers l'au-delà.

Le quatrième et avant-dernier paramètre d'apport africain à l'idéologie thanatologique se veut un constat. La mort: «une sanction que l'homme - et bien souvent la femme - a provoquée sciemment ou non par son comportement à l'égard du créateur » (Thomas 1984, 748). Évidemment, des mythes dogon et bambara (Griaule et Dieterlen 1965) au Mali, à l'Ouest du continent, jusqu'à ceux des Bàntu du Kàsaayì au cœur de l'Afrique au Congo R.D., "tous renvoient à une période pré-mythique, où l'être humain était immortel et où "Dieu dispensait abri et nourriture"; [...] tous évoquent un monde, enjeu de forces sans cesse en mouvement et qui viennent animer la vie, le pouvoir et la parole» (Fourche et Morlighen 2002). Et c'est par la faute de l'humain que Dieu, selon ces récits, a sanctionné celui-ci en lui infligeant la mort.

Par ailleurs, on ne peut pas ne pas mentionner les croyances et cérémonies qui ont trait à la mort en Égypte antique et qui étaient d'un impact vital. Inscrites dans le religieux, elles constituaient une grande «étape de la vie du pharaon, frère des dieux, qui devait après son décès vivre auprès des dieux un repos éternel. Les Égyptiens considéraient qu'après le décès, l'âme du défunt pouvait renaître et accéder au "royaume des morts" et au "repos éternel" " (Fourche et Morlighen 2002, 213-215). Deux étapes majeures composaient ce mythe égyptien de la mort. D'abord, «le voyage du défunt vers l'au-delà avec le rite d'embaumement». Ensuite son jugement par le dieu Osiris, au moment où il atteignait l'au-delà pour possiblement entrer au repos éternel. Ce rituel, bien détaillé dans le Livre des Morts du Scribe Negbed atteste que le corps humain, selon l'Égyptien, uni par plusieurs composantes dont le djet, l'équivalent du corps, et le $k a$, assimilé à l'esprit, vit, de la naissance à la mort, sous la guidance de ces deux noumènes. Au décès de l'homme, son entrée dans l'au-delà par le ka est conditionnée par l'embaumement du djet (le corps), rite qui fait renaître le défunt et lui donne accès au « royaume des morts et au repos éternel ». Au cas contraire, un djet non-embaumé change le ka en khat et, à partir de cet instant, l'accès 
au royaume et au repos immortel est quasiment impossible. Telle est la conviction qu'Osiris, instigateur de ce rite pour embaumer son épouse Isis en vue de lui redonner la vie, a imprimé dans cette donne. Dans le même ordre d'idées, sont mis dans le sarcophage à côté du défunt: offrandes, statuettes, et autres objets au titre «d'actants » pour lui tenir compagnie sur le chemin qui le conduit au jugement de l'âme. Cette réalité est également reproduite dans l'art architectural, en l'occurrence les pyramides. Évidemment, le chemin vers l'au-delà étant figuré, c'est par des couloirs hautement élevés que le défunt peut, de son lit, observer le ciel à partir de la chambre où il est déposé, permettant ainsi «à son âme de s'élever et d'atteindre le royaume des morts». En plus, un exemplaire du Livre des Morts était posé auprès du corps sans vie au titre du guide ou d'indicateur vers le royaume des Morts, en guise de préparation au jugement par les incantations et recueils constituant son contenu.

Enfin signalons, comme dernier apport, la pensée des Tropiques selon laquelle la vie ne finit pas. Elle est une continuité, même dans la mort. En Afrique, en effet, «la mort semble être, selon l'ethnologue Dominique Zahan, que nous citons encore, la conséquence inévitable de la vie» (Zahan 1979, 241). Elle est son prolongement avec l'au-delà comme son résultat inéluctable. C'est à ce titre que la mort constitue, d'après le Congolais Alphonse Ngindu Mushete, un passage d'une existence vers une autre existence. Ainsi, la vie de l'homme dans l'Au-delà est, pour ce théologien, la continuation de celle qu'il a passée sur la terre (1993). Pour faire court, nous affirmons, à la suite du philosophe sénégalais Souleymane Bachir Diagne (2000, 2), que «le muntu, l'être humain, est vivant et fort de ses liens à la divinité, à son clan, à sa famille, à ses descendants, comme il est fort et vivant de son patrimoine et de sa terre, de ce qu'elle porte et de ce qu'elle produit, de ce qui y pousse ou y vit.»

\section{Conclusion}

Après ces quelques réflexions, qu'il nous soit permis de conclure. Au terme de ce parcours sur vie et mort en Afrique Noire, nous avons conscience que nous n'avons pas épuisé un sujet si complexe. L'ayant, en plus, abordé dans un angle déterminé, d'autres aspects, peut-être plus importants, n'ont pas été développés. En guise de conclusion, nous retenons deux indications.

La première d'entre elles, nous l'empruntons chez Dominique Zahan qui, au cours de ses recherches a compris qu'au sujet du Continent Mère de l'humanité, il faut essayer de 
pénétrer dans l'âme noire afin d'y découvrir le principe animateur de la vie. [...] L'homme noir, microcosme où aboutissent, invisibles, d'innombrables fils qui tissent les choses et les lient entre eux, en vertu des règles de correspondance fournies par les catégories et les classifications. Il n'est pas le «Roi» de la création, mais plutôt l'élément central d'un système auquel il imprime une orientation centripète. (Zahan 1979, 14 et 16)

La seconde indication de conclusion est inscrite dans le registre cosmogonique africain. Il s'agit de la relation du coudoiement entre vie et mort. Évidemment, proches l'une de l'autre, dans la vision du monde africaine, vie et mort portent chaque existence. Elles la caractérisent. À ce titre, elles sont comme les deux faces de la main. Indissociables, vie et mort, en Afrique, sont liées alors qu'ailleurs dans d'autres cultures la mort jette le désarroi. On la cache, la maladie y compris. On n'en parle pas. Et il arrive souvent que sur la même rue ou sur le même pallier d'un immeuble, on n'apprenne le décès d'un voisin que plusieurs jours après son inhumation alors qu'on partageait le même lieu d'habitation. Par ailleurs, la vie, pour l'Africain, est donnée. Elle est un don et vient de quelque part. L'homme n'en est pas l'initiateur. Il n'en connait pas tous les secrets et elle reste une énigme. Ainsi en est-il également de la mort qui se trouve entourée du même mystère pour l'Africain. Alors que nombre de civilisations voient dans la mort une absurdité, un non-sens de l'existence humaine et que beaucoup, à l'idée de l'évoquer, perdent pieds, dépriment et parfois l'accélèrent en se suicidant, l'homme des Tropiques, lui, face à la culture de la mort envisage la vie, pense à la vie et cherche, à sa façon, comment l'augmenter si jamais elle diminuait. Ainsi, l'Afrique, si on veut rejoindre le père Engelbert Mveng qu'on a évoqué au début de cette étude, a plus à apporter et à dire dans ce débat à propos des deux terminus existentiels de l'itinéraire terrestre humain: la «vie» comme terminus a quo et la «mort» au titre de terminus ad quem ouvrant sur la vie. Une nouvelle vie. Une autre vie.

\section{Références}

Amaladoss, M. (2007), Jésus asiatique / trad. par A. Boutot, Paris, Presses de la renaissance.

Amougou, L.-B. (2008), «La mort dans les littératures africaines ", Faculté des Lettres et Sciences Humaines, Département d'Études Africaines, Université de Dschang (Cameroun) <lien internet indisponible>. 
Anganga, M. (2011), Analyse narrative du récit johannique de la Passion. Enjeux théologiques pour la célébration du Vendredi Saint, Villeneuve d'Ascq, A.N.R.T.

Aristote (1934), De l'âme (De Anima) / trad. par J. Tricot, Paris, Vrin.

Attolodé, L.-R.-B. (2004), L’idée de philosophie africaine. Séminaire de Formation des Formateurs sur l'appropriation des contenus des domaines de la "réflexion philosophique» et de la «vie sociale», Thiès: 16-19 février 2004, Dakar.

Braüer, G. (1994), «L'origine africaine de l'homme moderne », ANHK, 3, p. $132-150$.

Desroches Noblecourt, C. (2006), Le fabuleux héritage de l'Égypte, Paris, Pocket.

Diagne, S. B. (2000), «Revisiter "La Philosophie bantoue". L'idée d'une grammaire philosophique ", Philosophie et Politique en Afrique, 77, p. 44-53.

Diop, Cheik A. (1967), Antériorité des civilisations nègres - mythe ou vérité historique?, Paris, Présence Africaine.

(2007) [1984], «L'apport de l'Afrique au développement de la civilisation universelle ", Science et Philosophie. Textes 1960-1986, Université Cheik Anta Diop de Dakar, p. 7-191.

Diop, C. M'B. (2003), Cheikh Anta Diop, l'homme et l'œuvre, Présence africaine, Paris.

Hegel G. (1979), La Raison dans l'Histoire. Introduction à la Philosophie de l'Histoire / trad. par K. Papanioannou, Paris, Union Générale d'Éditions (10/18).

Hume, D. (1852), "Of National Characters", The Philosophical Works of David Hume, vol. 1, éd. par T.H. Green et T.H. Grosse, Londres.

Ogowè Nyalendo, I. (2010), «La victoire de la vie sur la mort », Overblog du 18 septembre.

Kabasele Lumbala, F. (2005), Renouer avec ses racines. Chemins d'inculturation, Paris, Karthala.

Kabasele Lumbala, F., Nkongolo, S. et Anganga, M.(2010), Naissances insolites en terre africaine, Paris, Karthala.

Kabuta, N.-S. (2008), Nkòngamyakù wa Cilubà - Mfwàlànsa (dictionnaire lubà-français), Gand, Éditions Recall-Ciyèm.

Lacarrière, J. (1998), Au coeur des mythologies, Gallimard, Paris. 
Marson, A. (1990), La physique aristotélicienne, Encyclopédie philosophique universelle, tome 1, vol. 2, Paris, P.U.F.

Mofila, R. (1997), Spiritualité pascale et concepts de "vie et mort" en Afrique. Essai de compréhension chez les Sours Disciples du Divin Maître, Mémoire de licence, Institut de Spiritualité africaine, Kinshasa.

Mouroux, J. (1943), Le sens chrétien de l'homme, Paris, Aubier.

Mujinya Nimisi, C.-E. (1978), L’homme dans l'univers des bantu, $2^{\mathrm{e}}$ éd., Kinshasa, Presses universitaires du Zaïre.

Mulago, V. (1973), La religion traditionnelle des bantu et leur vision du monde, Kinshasa, Presses universitaires du Zaïre.

Mveng, E. et Lipawing, B. -L. (1996), Théologie, Libération et Cultures Africaines, Paris, Clé (Présence Africaine).

Obenga, T. (1997-1998), "Anthropologie pharaonique. Texte à l'appui ", ANHK, 6-7, p. 9-53.

(1994), "Origine commune de l'Égypte ancien, du copte et des langues Négro-Africaines », ANKH, 3, p. 115-135.

ОмотUnde, P. (2000), L’origine négro-africaine du savoir grec, Yaoundé, Édition Menaibuc (Connaissance du Monde nègre).

Platon (1950), Euvres complètes, tome 1, Phédon, Paris, Gallimard (La pléiade).

SHENOC (2008), «La conception des peuples noirs de la mort et de l'immortalité ", <http://www.shenoc.com/la_conception_des_peuples_noirs_ de_la_mort_et_de_l-immortalite.htm>.

Tempels, P., La philosophie Bantoue, (Présence Africaine), Paris, Clé, 1945.

Thомas, L.-V. (1968), Cinq essais sur la mort africaine, Université de Dakar, Faculté des lettres et sciences humaines. (1982), La mort africaine. Idéologie funéraire en Afrique Noire, Paris, Payot.

Tresmontant, C. (1971), Le problème de l'âme, Seuil, Paris.

Wigoder, G. (1993), "Mort», in Dictionnaire Encyclopédique du Judaïsme, Paris, Cerf, p. 773, col.1.

Zahan, D. (1970), Religion, spiritualité et pensée africaine, Paris, Payot. (1979), «Essai sur les mythes africains d'origine de la mort ", L’homme, 9/4, p. 41-50. 


\section{Résumé}

En Afrique, continent-Mère de l'homme et source de notre civilisation, vie et mort, depuis plus de 200000 ans avant notre ère, sont liées. Inséparables. Elles constituent, ensemble, les deux faces de l'existence humaine et, par ce fait, la mort se veut la conséquence de la vie. Dès lors, dans la cosmogonie négro-africaine dont les traces sont visibles dans le judaïsme et le christianisme, l'idéologie de la vie prime sur celle de la thanatologie, car la vie ne finit pas avec la mort. A contrario, elle la dépasse, la transcende et continue dans l'Au-delà. Ainsi, la mort n'est pas le dernier mot de la vie pour l'Africain. Celle-ci est, reste et demeurera une phrase en pointillés qui s'achèvera au village des ancêtres lors du retour final.

\footnotetext{
Abstract

In Africa, motherland of humanity and background of our civilization, life and death have been closely related for over 200000 years. Inseparable, even. Together, they constitute the two sides of human existence and by this fact, death is seen as the consequence of life. As such, in negro-African cosmogony of which traces can be found in Judaism and Christianity, the ideology of life primes over thanatology, since life doesn't end with death. To the contrary, life goes beyond death, transcends it and continues into the Afterlife. Therefore, for the Africans, death is not life's last word. It is and stays an open-ended sentence that culminates in our ancestors' village at the moment of the final return.
} 